\title{
Pump Spectral Bandwidth, Birefringence, and Entanglement in Type-II Parametric Down Conversion
}

\author{
Daniel Erenso \\ Department of Physics \& Astronomy, Middle Tennessee State University, Murfreesboro, TN 37 132, USA \\ Correspondence should be addressed to Daniel Erenso, derenso@mtsu.edu \\ Received 15 April 2009; Accepted 1 June 2009 \\ Recommended by ortunato Tito Arecchi
}

\begin{abstract}
The twin photons produced by a type-II spontaneous parametric down conversion are well know as a potential source of photons for quantum teleportation due to the strong entanglement in polarization. This strong entanglement in polarization, however, depends on the spectral composition of the pump photon and the nature of optical isotropy of the crystal. By exact numerical calculation of the concurrence, we have shown that how pump photons spectral width and the birefringence nature of the crystal directly affect the degree of polarization entanglement of the twin photons.
\end{abstract}

Copyright ( $\odot 2009$ Daniel Erenso. This is an open access article distributed under the Creative Commons Attribution License, which permits unrestricted use, distribution, and reproduction in any medium, provided the original work is properly cited.

The realization of faithful transmission of information in quantum communication $[1,2]$, completely secure information exchange in quantum cryptography [3], and the fastest quantum computers $[4,5]$ fundamentally requires entangled state of the Bell type. The twin photons produced by spontaneous parametric down conversion (SPDC) via a pump photon interacting with a $\beta$-barium borate, $\mathrm{BaB}_{2} \mathrm{O}_{4}$ (BBO), nonlinear crystal are one of the common source of entangled state of the Bell type. There is an increasing interest in the spectral and spatial properties of the down-converted photons and, specifically, in achieving particular spatial and spectral profiles $[6,7]$. The spectral and spatial characteristics of SPDC photons are influenced both by the phase-matching properties of the crystal and by the spectral composition of the pump light. Previous works have shown that a broadband pump, for example, has been shown to lead to different signal and idler spectra in type-II SPDC [8]. Recently, Humble and Grice [9] using the signal-idler state in type-II SPDC have reported that polarization entanglement of the signal and idler photons as well as the fidelity of the teleportation based on the polarization states of these photons depends on the spectral entanglement. This study is carried out for the case in which the amplitudes in the joint signal-idler state which determines the spectral entanglement is approximated by a correlated Gaussian function. In this study we use the exact joint signal-idler biphoton state in type-II SPDC [8] and investigate how the degree of polarization entanglement of the two photons changes as the corresponding crystal transit times and the pump spectral composition vary. These results are also discussed in comparison with the approximate results reported by Humble and Grice [9].

In parametric down-conversion type-I phase matching leads to two photons with same polarization. In this phase matching, if the down-conversion is degenerated, a pair of two photons with identical frequency emerges on a cone [10] which is centered on the pump beam whose opening angle depends on the angle between the crystal optic axis and the pump. On the other hand with type-II phase matching, the down-converted photons are emitted into two cones, one horizontally polarized $(h)$ and the other vertically polarized $(v)$. In the collinear situation the two cones are tangent to one another on exactly one line, namely, the pump beam direction. The two particular directions can be chosen in the noncollinear case where the two cones intersect each other, and the two correlated photons are produced in an entangled state of the form, $|h, v\rangle+|v, h\rangle$. Experimentally, such form of polarization-entangled state using this technique has been demonstrated using a single BBO crystal [11].

In Type-II parametric down-conversion we consider the case where the nonlinearity of the crystal does not depend on frequency so that the gain of the parametric interaction is constant. We also assume that the down-converted beams 
are constrained to be nearly collinear. The beam that pumps the crystals is pulsed laser which is much stronger than the down-converted beams. Under these conditions and taking into account that parametric down-conversion is very much ineffective, along the two directions, the state of the two photons can be written as $[8,12]$

$$
\begin{aligned}
& \left|\psi_{23}\right\rangle \\
& =A \int d \omega_{2} \int d \omega_{3} \alpha\left(\omega_{2}+\omega_{3}\right)\left\{\Phi\left(\omega_{2}, \omega_{3}\right)\right. \\
& \times\left|h_{2}\left(\omega_{2}\right), v_{3}\left(\omega_{3}\right)\right\rangle \\
& \left.+\Phi\left(\omega_{3}, \omega_{2}\right)\left|h_{3}\left(\omega_{3}\right), v_{2}\left(\omega_{2}\right)\right\rangle\right\}
\end{aligned}
$$

where $A$ is a normalization constant,

$$
\alpha\left(\omega_{i}+\omega_{j}\right)=\exp \left[-\left(2 \omega_{0}-\left(\omega_{i}+\omega_{j}\right)\right)^{2} / 2 \sigma_{p}^{2}\right]
$$

is the pump envelope function which ensures conservation of energy, and

$$
\Phi\left(\omega_{i}, \omega_{j}\right)=\frac{\sin \left\{\left[k_{2}\left(\omega_{i}\right)+k_{3}\left(\omega_{j}\right)-k_{p}\left(\omega_{i}+\omega_{j}\right)\right] L\right\}}{\left[k_{2}\left(\omega_{i}\right)+k_{3}\left(\omega_{j}\right)-k_{p}\left(\omega_{i}+\omega_{j}\right)\right] L}
$$

is the phase-matching function which ensures conservation of momentum for $i, j=2,3$ and $i \neq j$. Here, $\sigma_{p}$ determines the bandwidth of the pump photons. Since the pump envelope function depends on the sum frequency, it is symmetric with respect to $\omega_{2}$ and $\omega_{3}$. However, in the phase-matching function $\Phi\left(\omega_{i}, \omega_{j}\right)$, since the crystal is birefringent $k_{2}\left(\omega_{i}\right) \neq k_{3}\left(\omega_{j}\right), \Phi\left(\omega_{i}, \omega_{j}\right)$ is not symmetric in its frequency arguments $\left(\Phi\left(\omega_{j}, \omega_{i}\right) \neq \Phi\left(\omega_{i}, \omega_{j}\right)\right)$. By expanding the three wave vectors in series about their respective center frequencies (i.e., $k_{2}\left(\omega_{i}\right)$ and $k_{3}\left(\omega_{j}\right)$ about $\omega_{0}, k_{p}\left(\omega_{i}+\omega_{j}\right)$ about $\left.2 \omega_{0}\right)$, for a perfect phase matching at the center frequencies (i.e., $k_{2}\left(\omega_{0}\right)+k_{3}\left(\omega_{0}\right)=k_{p}\left(2 \omega_{0}\right)$ ) and small mismatch for off-central frequencies, the phase matching function can be approximated as

$$
\Phi\left(\omega_{i}, \omega_{j}\right) \simeq \frac{\sin \left[\tau_{p 2}\left(\omega_{i}-\omega_{0}\right)+\tau_{p 3}\left(\omega_{j}-\omega_{0}\right)\right]}{\tau_{p 2}\left(\omega_{i}-\omega_{0}\right)+\tau_{p 3}\left(\omega_{j}-\omega_{0}\right)},
$$

where the constants

$$
\tau_{p n}=\left(\partial k_{p} /\left.\partial \omega\right|_{\omega=2 \omega_{0}}-\partial k_{n} /\left.\partial \omega\right|_{\omega=\omega_{0}}\right) L
$$

represent the difference in transit times through the crystal between the pump and the first down-converted photon $(n=$ 2 ) and the pump and the second down-converted photon $(n=3)$, respectively.
Introducing the variables, $\Delta \omega_{i, j}=\omega_{i, j}-\omega_{0}$, and using the expressions for the phase-matching function in (4) and the pump envelop function in (2), we can write (1) as

$$
\begin{aligned}
& \left|\psi_{23}\right\rangle \\
& =\int d \Delta \omega_{2} \int d \Delta \omega_{3}\left\{f\left(\Delta \omega_{i}, \Delta \omega_{j}\right)\right. \\
& \times\left|h_{2}\left(\Delta \omega_{i}\right), v_{3}\left(\Delta \omega_{j}\right)\right\rangle \\
& \left.+f\left(\Delta \omega_{j}, \Delta \omega_{i}\right)\left|h_{3}\left(\Delta \omega_{j}\right), v_{2}\left(\Delta \omega_{i}\right)\right\rangle\right\},
\end{aligned}
$$

where the function $f\left(\Delta \omega_{i}, \Delta \omega_{j}\right)$ is given by

$$
\begin{aligned}
& f\left(\Delta \omega_{i}, \Delta \omega_{j}\right)= N_{i j} \exp \left\{-\left(\Delta \omega_{i}+\Delta \omega_{j}\right)^{2} / 2 \sigma_{p}^{2}\right\} \\
& \times\left\{\sin \left[\tau_{p 2} \Delta \omega_{i}+\tau_{p 3} \Delta \omega_{j}\right]\right. \\
&\left./\left(\tau_{p 2} \Delta \omega_{i}+\tau_{p 3} \Delta \omega_{j}\right)\right\}
\end{aligned}
$$

for $i=2,3$ with $i \neq j$, representing the spectral amplitude for the two different polarization states of the down-converted photons. These amplitudes depend on the bandwidth of the pump mode and crystal transit times $\left(\tau_{p 2}\right.$ and $\left.\tau_{p 3}\right)$ for the down-converted photon 2 and photon 3 with respect to the transit time for the pump mode, respectively. Using (6) and (7), we will study explicitly the direct effect of the transit times difference $\left(\tau_{p 2}, \tau_{p 3}\right)$ and the pump spectral linewidth $\left(\sigma_{p}\right)$ in the degree of the polarization entanglement between the two down-converted photons.

The degree of entanglement of a bipartite system, quantitatively, can be described using the von Neumann entropy $[13,14]$ or the concurrence [15]. Here, we will use the concurrence to quantitatively describe the entanglement of the two photons. To this end, for the case of discrete bipartite density matrix $\rho$, the concurrence, $C$, is given by [15]

$$
C=\max \left\{0, \lambda_{1}-\lambda_{2}-\lambda_{3}-\lambda_{4}\right\}
$$

in which the $\lambda_{k}^{\prime}$ s (with $\lambda_{1} \geqq \lambda_{2} \geqq \lambda_{3} \geqq \lambda_{4}$ ) are the square roots of the eigenvalues of the Hermitian matrix

$$
\tilde{\rho}=\hat{\rho}_{23} \hat{\sigma}_{2 y} \otimes \hat{\sigma}_{3 y} \hat{\rho}_{23}^{*} \hat{\sigma}_{3 y} \otimes \hat{\sigma}_{2 y} .
$$

In (9), $\hat{\rho}_{23}=\left|\psi_{23}\right\rangle\left\langle\psi_{23}\right|$ is the two photon density operator which can be expressed as a quadruple integral using the state vector in (6), $\hat{\sigma}_{2 y}=i\left|v_{2}\right\rangle\left\langle h_{2}|-i| h_{2}\right\rangle\left\langle v_{2}\right|$ and $\hat{\sigma}_{3 y}=$ $i\left|v_{3}\right\rangle\left\langle h_{3}|-i| h_{3}\right\rangle\left\langle v_{3}\right|$ are the Pauli single-qubit operators. By tracing over the spectral states of the two photons, we determined the matrix elements of the Hermitian matrix, $\tilde{\rho}$. We then diagonalized the matrix to obtain the eigenvalues $\lambda_{1}, \lambda_{2}, \lambda_{3}$, and $\lambda_{4}$ that we substituted into (8). The resulting expression for the concurrence is found to be

$$
C=\int d\left(\Delta \omega_{2}\right) d\left(\Delta \omega_{3}\right) f\left(\Delta \omega_{2}, \Delta \omega_{3}\right) f^{*}\left(\Delta \omega_{3}, \Delta \omega_{2}\right) .
$$


Equation (10) depends on the spectral amplitudes $f\left(\Delta \omega_{2}\right.$, $\left.\Delta \omega_{3}\right)$ and $f\left(\Delta \omega_{3}, \Delta \omega_{2}\right)$ which are determined by the birefringence of the crystal and the pump spectral distribution. If the crystal is optically isotropic, we would find that $f\left(\Delta \omega_{2}, \Delta \omega_{3}\right)=f\left(\Delta \omega_{3}, \Delta \omega_{2}\right)$ and the concurrence, for normalized spectral amplitudes

$$
\int d\left(\Delta \omega_{\mathrm{i}}\right) d\left(\Delta \omega_{\mathrm{j}}\right)\left|f\left(\Delta \omega_{\mathrm{i}}, \Delta \omega_{\mathrm{j}}\right)\right|^{2}=1,
$$

become $C=1$. However, when $f\left(\Delta \omega_{2}, \Delta \omega_{3}\right) \neq f\left(\Delta \omega_{3}, \Delta \omega_{2}\right)$, previous studies have shown that the degree of polarization entanglement can drastically decrease [9]. These studies assume an approximate Gaussian function for the Sinc function in (4) describing the phase mismatch resulting from optical anisotropy of the crystal which leads to different relative transit times for the down-converted photons $\left(\tau_{p 2}\right.$ and $\left.\tau_{p 3}\right)$. In the following, without this approximation, we will study the direct effect of optical anisotropy of the crystal and the spectral line width of a Gaussian pump photons. To this end, we note that applying the expression for the spectral amplitudes in (7), the concurrence in (10) can be rewritten as

$$
\begin{aligned}
C= & N_{23} N_{32} \int d\left(\Delta \omega_{2}\right) d\left(\Delta \omega_{3}\right) \exp \left[-\left(\Delta \omega_{2}+\Delta \omega_{3}\right)^{2} / \sigma_{p}^{2}\right] \\
& \times \sin \left[\tau_{p 2} \Delta \omega_{2}+\tau_{p 3} \Delta \omega_{3}\right] \sin \left[\tau_{p 2} \Delta \omega_{3}+\tau_{p 3} \Delta \omega_{2}\right] \\
& /\left(\tau_{p 2} \Delta \omega_{2}+\tau_{p 3} \Delta \omega_{3}\right)\left(\tau_{p 2} \Delta \omega_{3}+\tau_{p 3} \Delta \omega_{2}\right),
\end{aligned}
$$

where the constants $N_{i j}$ 's are normalization constants that should be obtained using (11).

We first examine how the degree of the entanglement of the down-converted photons depends on the spectral bandwidth of the pump photons, $\sigma_{p}$. In Figure 1, on the inset, we have shown the square of the magnitude of the Gaussian spectral profile of the pump photons $\left[\exp \left\{-\left(\Delta \omega_{2}+\Delta \omega_{3}\right)^{2} / \sigma_{p}^{2}\right\}\right]$ for different values of the pump bandwidth, $\sigma_{p}=0.1$ (blue [a]), 0.5 (red [b]), 1.0 (green [c]), and 4.0 (pink [d]), respectively. For each of these values of spectral bandwidth the concurrence in (12) along with corresponding normalization factors $N_{i j}$ 's in (11) is calculated as a function of crystal transit time difference between the pump and the down-converted photons, $\tau_{p 2}$ and $\tau_{p 3}$. The colored curves shown in Figure 1 is the result for the corresponding concurrence, $C$, as function of $\tau_{p 2} / \tau_{p 3}$. These curves labeled as (a), (b), (c), and (d) show how the concurrence is affected as we vary $\tau_{p 2} / \tau_{p 3}$ when the pump photons bandwidth is $\sigma_{p}=0.1$ [blue (a)], 0.5 [red (b)], 1.0 [green (c)], and 4.0 [pink (d)], respectively. Comparing these curves with the corresponding pump photons spectral profile, we note that as the pump bandwidth becomes narrower, the effect of the difference in crystal transit time on the entanglement of the two photons becomes negligible. This means that the optical anisotropy of the crystal which causes the difference in crystal transit times has no significant effect on the entanglement when the incident pump photons have a negligible bandwidth. On the other
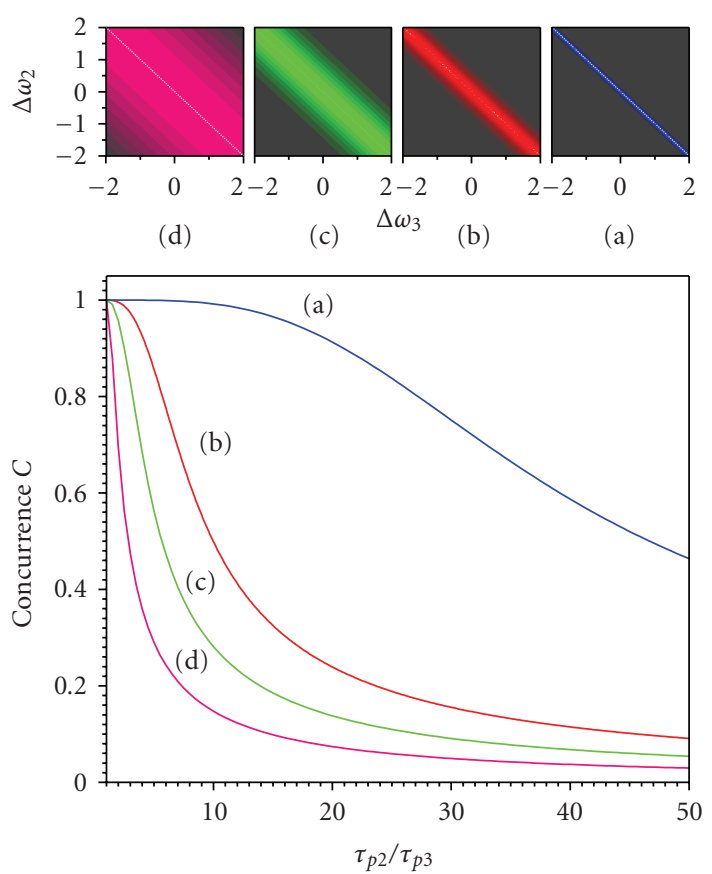

FIGURE 1: The concurrence as a function of the ratio of the crystal transit times difference $\left(\tau_{p 2} / \tau_{p 3}\right)$ and the pump and the second down-converted photon. Note: $\sigma_{p}=0.1$ (blue [a]), $\sigma_{p}=0.5$ (red $[\mathrm{b}]), \sigma_{p}=1.0$ (green [c]), and $\sigma_{p}=4.0(\operatorname{pink}[\mathrm{d}])$.

hand, as the pump spectral profile becomes broader, the effect of optical anisotropy of the crystal becomes significant. We observe this behavior for the entanglement in Figure 1 where the concurrence becomes less than unity, rapidly, as the pump becomes broader and $\tau_{p 2} / \tau_{p 3}<1$. However, when $\tau_{p 2} / \tau_{p 3}=1$, the two down-converted photons have the same crystal transit time which means that the crystal is optically isotropic. Under this condition, independent of the spectral bandwidth of the pump photons, the entanglement remains maximal, $C=1$.

Humble and Grice [9] have studied the entanglement of the two photons when the expression for the joint spectral amplitude, $f\left(\Delta \omega_{1}, \Delta \omega_{2}\right)$, in (7) is approximated by choosing a constant $\gamma=0.42$ to match the full width at half maximum of a Gaussian function for the phase matching function:

$$
\begin{gathered}
\sin \left[\left(\tau_{p 2} \Delta \omega_{2}+\tau_{p 3} \Delta \omega_{3}\right)\right] / \tau_{p 2} \Delta \omega_{2}+\tau_{p 3} \Delta \omega_{3} \\
\simeq \exp \left(-\frac{\gamma}{2}\left(\tau_{p 2} \Delta \omega_{2}+\tau_{p 3} \Delta \omega_{3}\right)^{2}\right),
\end{gathered}
$$

so that joint spectral amplitude in (7) can be approximated as

$$
\begin{aligned}
f\left(\Delta \omega_{2}, \Delta \omega_{3}\right) \simeq N_{23} \exp [ & -\left(\Delta \omega_{2}+\Delta \omega_{3}\right)^{2} / 2 \sigma_{p}^{2} \\
& \left.-\gamma\left(\tau_{p 2} \Delta \omega_{2}+\tau_{p 3} \Delta \omega_{3}\right)^{2} / 2\right] .
\end{aligned}
$$




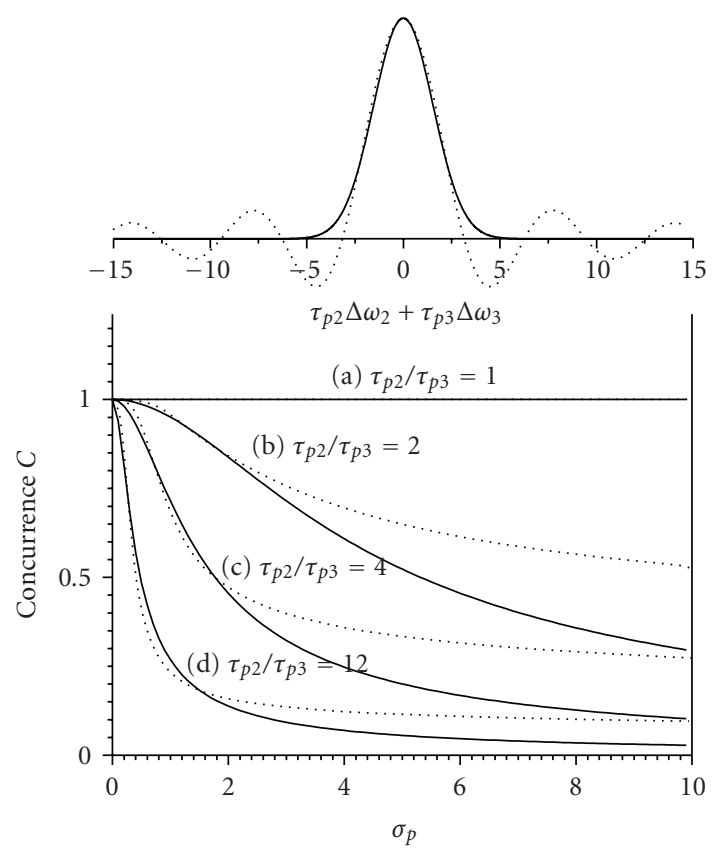

Figure 2: The concurrence as a function of the pump spectral width $\sigma_{p}$ for different values of the ratio of the crystal transit times $\left(\tau_{p 2} / \tau_{p 3}\right)$. The dotted curves are the exact, and the solid curves are approximate. The in-set on the top represents the phase-matching functions, exact (the dotted curve) and approximate (the solid curve).

Then, for this approximation, the expression for the concurrence in (10) becomes

$$
C=\sqrt{4 \sigma_{2}^{2} \sigma_{3}^{2}\left(1-\rho^{2}\right) /\left[\left(\sigma_{2}^{2}+\sigma_{3}^{2}\right)^{2}-\sigma_{2}^{2} \sigma_{3}^{2} \rho^{2}\right]},
$$

where

$$
\begin{gathered}
\rho=-\left(1-\gamma \tau_{p 2} \tau_{p 3} \sigma_{p}^{2}\right) / \sqrt{\left(1+\gamma \tau_{p 2}^{2} \sigma_{p}^{2}\right)\left(1+\gamma \tau_{p 3}^{2} \sigma_{p}^{2}\right)}, \\
\sigma_{2}^{-2}=\left(\sigma_{p}^{-2}+\gamma \tau_{p 2}^{2}\right)\left(1-\rho^{2}\right), \quad \sigma_{3}^{-2}=\left(\sigma_{p}^{-2}+\gamma \tau_{p 3}^{2}\right)\left(1-\rho^{2}\right) .
\end{gathered}
$$

Using the results in (15)-(16), we have analyzed the entanglement of the two photons in comparison with the exact result as predicted by (12). The concurrence, $C$, for both the exact and approximate cases is calculated for selected values of $\tau_{p 2} / \tau_{p 3}$ as a function of the pump spectral width, $\sigma_{p}$, and the results are shown in Figure 2. When the crystal is optically isotropic $\left(\tau_{p 2} / \tau_{p 3}=1\right)$, the photons reveal a maximal entanglement $(C=1)$ independent of the pump spectral width since the phase-matching function becomes symmetrical. As a result the concurrence is equal to unity for all values of the spectral width for both the exact $(\operatorname{sinc})$ and approximate (Gaussian) phase-matching functions as described by the overlapping solid and dotted curves; see (a) in Figure 2. On the other hand as the crystal becomes optically anisotropic $\left(\tau_{p 2} / \tau_{p 3}>1\right)$, the approximate and the exact results for the concurrence begin to differ when the spectral width of the pump photons increases, as illustrated by the solid and dotted curves (b)-(d) in Figure 2, respectively. These curves show that the approximate results under-predict the degree of entanglement of the downconverted photons for spectrally broad Gaussian pump photons as compared to the corresponding exact prediction. This is shown by the solid curves (approximate) which stay under the corresponding dotted curves representing the exact result for the concurrence.

In conclusion, the Gaussian approximation for the phase matching function in type-II SPDC does not accurately predict the degree of polarization entanglement of the downconverted photons for a pump photon with broad spectral composition. This result may potentially be usefully to design a more effective experimental scheme for optimization of polarization entanglement of the twin photons in type-II SPDC.

\section{Acknowledgments}

This work is in part supported by US Department of Energy and Oak Ridge Institute for Science \& Education and Middle Tennessee State University. The author thanks Travis S. Humble and Warren P. Grice.

\section{References}

[1] C. H. Bennett, G. Brassard, C. Crépeau, R. Jozsa, A. Peres, and W. K. Wootters, "Teleporting an unknown quantum state via dual classical and Einstein-Podolsky-Rosen channels," Physical Review Letters, vol. 70, no. 13, pp. 1895-1899, 1993.

[2] N. Gisin and R. Thew, "Quantum communication," Nature Photonics, vol. 1, no. 3, pp. 165-171, 2007.

[3] A. K. Ekert, "Quantum cryptography based on Bell's theorem," Physical Review Letters, vol. 67, no. 6, pp. 661-663, 1991.

[4] M. A. Nielsen and I. L. Chuang, Quantum Computation and Quantum Information, Cambridge University Press, New York, NY, USA, 2000.

[5] E. Knill, R. Laflamme, and G. J. Milburn, "A scheme for efficient quantum computation with linear optics," Nature, vol. 409, no. 6816, pp. 46-52, 2001.

[6] S. Carrasco, M. B. Nasr, A. V. Sergienko, et al., "Broadband light generation by noncollinear parametric downconversion," Optics Letters, vol. 31, no. 2, pp. 253-255, 2006.

[7] K. A. O'Donnell and A. B. U'Ren, "Observation of ultrabroadband, beamlike parametric downconversion," Optics Letters, vol. 32, no. 7, pp. 817-819, 2007.

[8] W. P. Grice and I. A. Walmsley, "Spectral information and distinguishability in type-II down-conversion with a broadband pump," Physical Review A, vol. 56, no. 2, pp. 1627-1634, 1997.

[9] T. S. Humble and W. P. Grice, "Spectral effects in quantum teleportation," Physical Review A, vol. 75, no. 2, Article ID 022307, 2007.

[10] R. Y. Chiao, P. G. Kwiat, and A. M. Steinberg, "Optical tests of quantum mechanics," in Advances in Atomic, Molecular and Optical Physics, B. Bederson and H. Walther, Eds., vol. 34, Academic Press, New York, NY, USA, 1994.

[11] P. G. Kwiat, K. Mattle, H. Weinfurter, A. Zeilinger, A. V. Sergienko, and Y. Shih, "New high-intensity source of polarization-entangled photon pairs," Physical Review Letters, vol. 75, no. 24, pp. 4337-4341, 1995. 
[12] T. E. Keller and M. H. Rubin, "Theory of two-photon entanglement for spontaneous parametric down-conversion driven by a narrow pump pulse," Physical Review A, vol. 56, no. 2, pp. 1534-1541, 1997.

[13] B. Schumacher, "Quantum coding," Physical Review A, vol. 51, no. 4, pp. 2738-2747, 1995.

[14] R. Jozsa and B. Schumacher, "A new proof of the quantum noiseless coding theorem," Journal of Modern Optics, vol. 41, pp. 2343-2349, 1994.

[15] W. K. Wootters, "Entanglement of formation of an arbitrary state of two qubits," Physical Review Letters, vol. 80, no. 10, pp. 2245-2248, 1998. 

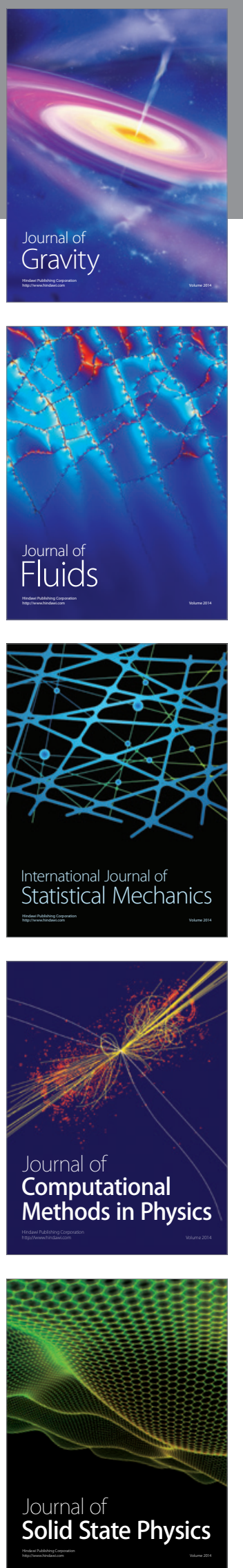

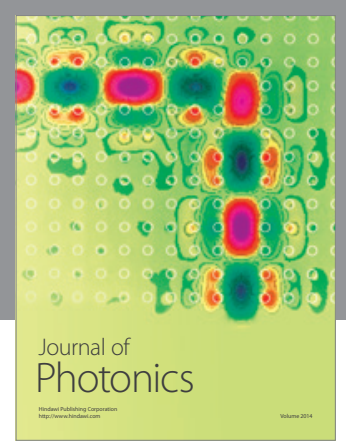

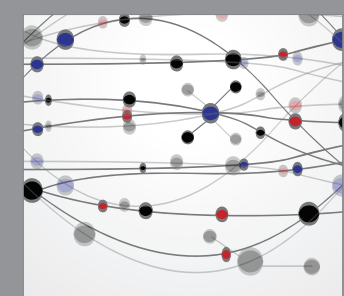

The Scientific World Journal
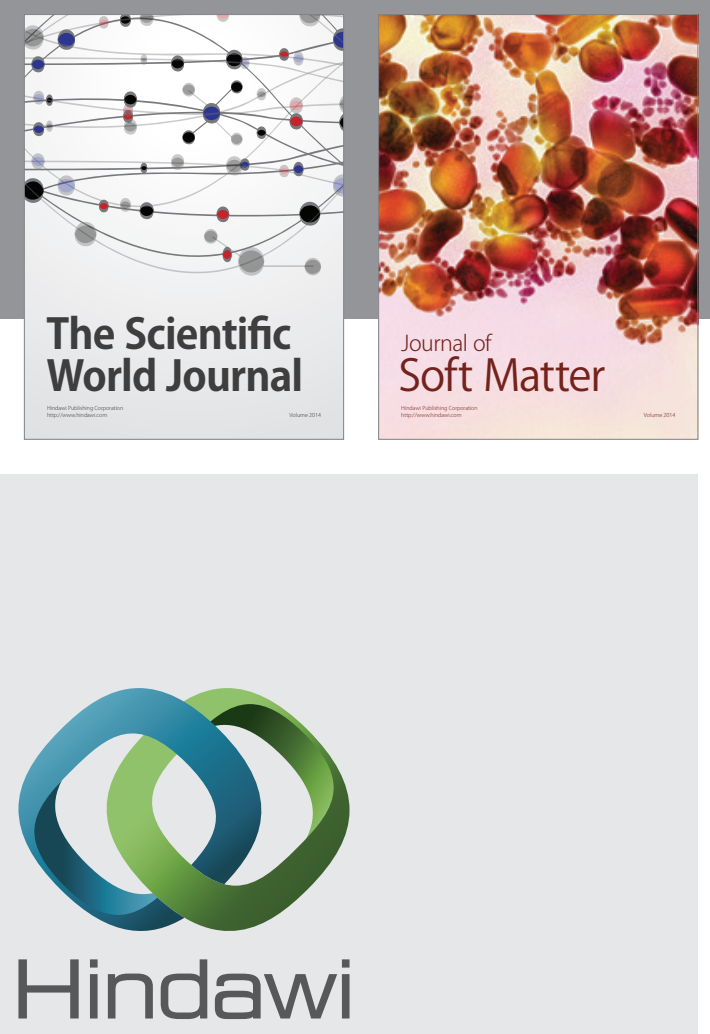

Submit your manuscripts at

http://www.hindawi.com
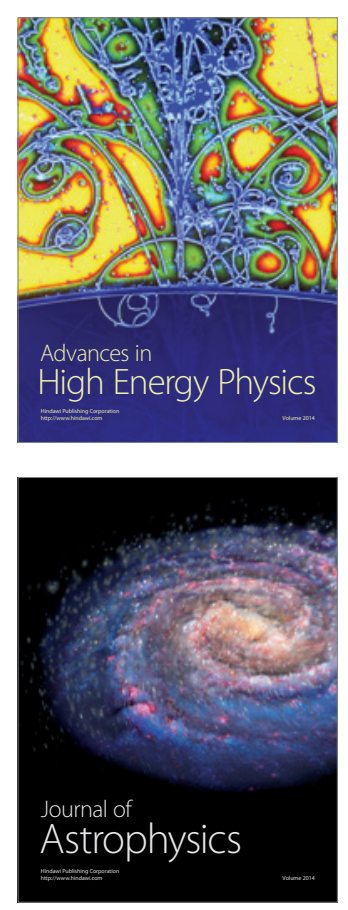
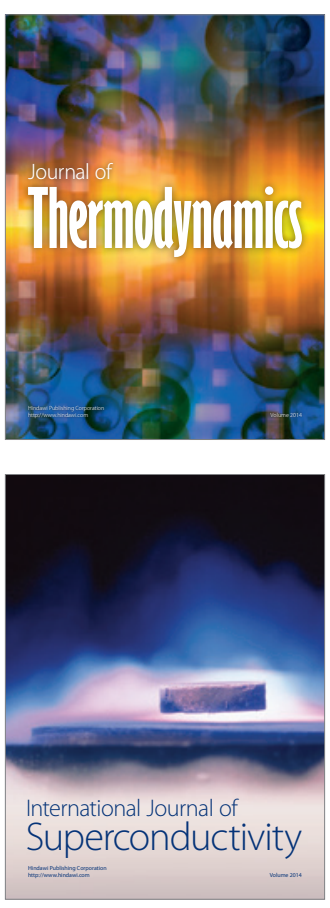
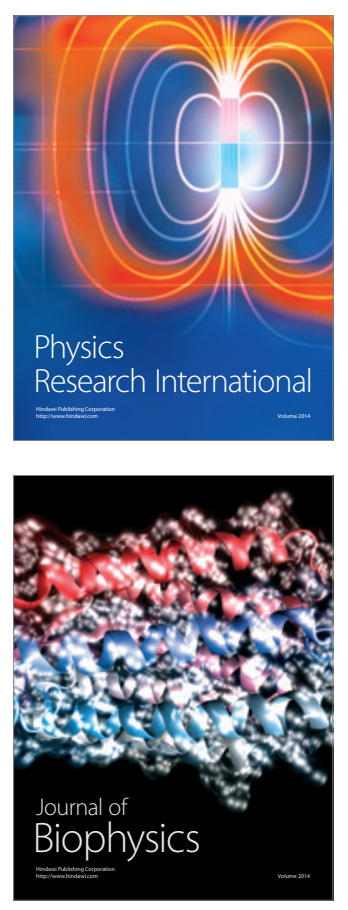
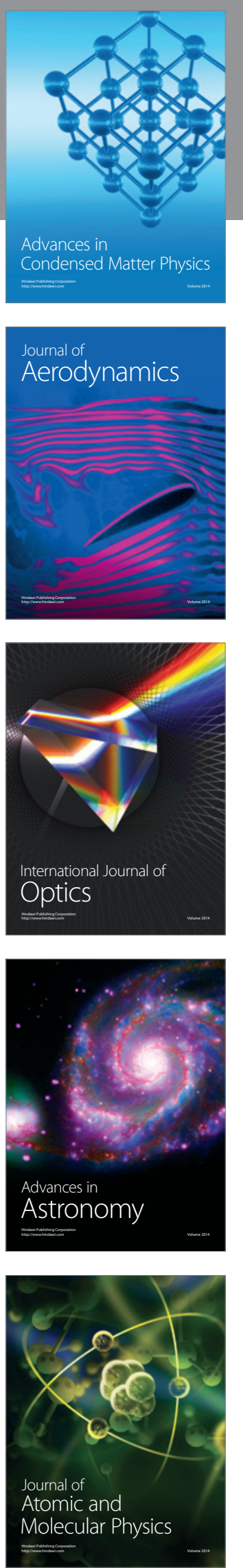